EXTENDED REPORT

\title{
Optical coherence tomography guided retreatment of photodynamic therapy
}

\author{
I Krebs, S Binder, U Stolba, K Schmid, C Glittenberg, W Brannath, A Goll
}

Br J Ophthalmol 2005;89:1184-1187. doi: 10.1136/bjo.2005.067389

See end of article for authors' affiliations

.....................

Correspondence to: Dr I Krebs, Department of Ophthalmology, Rudolf Foundation Clinic, Juchgasse 25, 1030 Vienna, Austria; Ilse. Krebs@wienkav.at

Accepted for publication 21 April 2005
Aim: To evaluate the results of a retreatment modality of photodynamic therapy (PDT) based on optical coherence tomography (OCT) and fluorescein angiography (FA). To quantify the effect of PDT with the help of measurement of the retinal thickness.

Methods: Eyes with predominantly classic subfoveal choroidal neovascularisation (CNV) due to age related macular degeneration were included. PDT was performed every three months, when needed. OCT, FA, and measures of distance acuity were performed at baseline, after 6 weeks, 3 months, and from then on every 3 months. A control group of a consecutive series of eyes that had been retreated based only on FA results was installed.

Results: Forty eyes of 38 patients were included. The average age was 73 years. The maximum retinal thickness decreased from $404 \mu \mathrm{m}$ at baseline to $281.6 \mu \mathrm{m}$ at month 12 . Furthermore there was a significant decrease of retinal thickness in both subgroups. The number of retreatments was reduced, when activity was diagnosed using OCT and FA. $(2.4 v 4.0)$. The distance acuity correlated significantly with the maximum retinal thickness $(p=0.0042)$.

Conclusion: Information about the activity of a neovascular lesion can be obtained with the help of OCT. The retreatment modalities can be optimised by using OCT and FA and the number of retreatments can be reduced.
$\mathrm{P}$ hotodynamic therapy (PDT) is a new therapeutic option in the therapy of choroidal neovascularisations (CNV) of different aetiology. ${ }^{1-3}$ The efficacy of PDT in cases of CNV due to age related macular degeneration has been proven in randomised multicentre clinical trials $\left(\mathrm{TAP}^{12}\right.$ ). The eligibility for this treatment is based on fluorescein angiographic findings. Optical coherence tomography (OCT) is, compared with fluorescein angiography (FA), a non-invasive and noncontact imaging modality producing cross sectional images of retinal tissues. ${ }^{4}$ OCT provides an objective test for quantitative evaluations of the retinal thickness. ${ }^{5}$ In the FA the amount of retinal oedema can only be estimated, based on the amount of leakage. In our study, we examined whether it is possible to quantify the effect of PDT with the help of OCT. Retreatment was based on FA and OCT findings. The results were compared with the results of a consecutive series of eyes that had been retreated only based on the results of FA.

\section{PATIENTS AND METHODS}

Between April 2000 and June 2002 eyes with subfoveal predominantly classic CNV as a result of age related macular degeneration, ${ }^{6}$ that had been treated with PDT, were enrolled in our study.

PDT was performed according to the $\mathrm{TAP}^{1}$ guidelines. The intravenous infusion of $6 \mathrm{mg} / \mathrm{m}^{2}$ body surface area of verteporfin (Novartis) was followed by irradiation 15 minutes after the start of sensitiser administration, with light at $689 \mathrm{~nm}$, delivered by an ocular photoactivation diode and laser linked slit lamp (Coherent Inc, Palo Alto, CA, USA). The spot size was $1000 \mu \mathrm{m}$ larger than the greatest diameter of the lesion measured in FA. Retreatment was performed every three months if any activity was seen in FA and OCT (subgroup 1). A control group of a consecutive series of 12 eyes treated after November 2001 was installed (subgroup 2). These eyes were treated if any leakage in the late phase angiograms could be seen. Signs, obtained with the OCT, of an active leaking membrane needing retreatment were:
1. detachment of the neurosensory retina;

2. increase in retinal thickness;

3. retinal thickness greater than $250 \mu \mathrm{m}$; and

4. fibrovascular detachment of the retinal pigment epithelium (RPE).

An OCT scan and FA images of an eye showing signs of activity is presented in line 1 of figure 1

At baseline (examination 0: between 1 and 7 days before treatment) and the follow up (6 weeks: examination 1, 3 months: examination 2, and then continuing every 3 months after treatment: examination 3, 4, 5, ..) the following examinations were performed: best corrected distance acuity tested with standard ETDRS charts, ${ }^{7}$ FA, and OCT.

A decrease in distance acuity of more than one line was counted as visual loss. Deterioration or improvement of one line or less was defined as unchanged. Increase of distance acuity of more than one line was called improvement.

FA and the measurement of the lesion size were performed with the Heidelberg retina angiograph (Heidelberg Engineering, Heidelberg, Germany). Six to eight pictures were taken in the early phase after injection, then every 15 seconds, after 1 minute, after 2 minutes, and finally late pictures after 5 and 10 minutes.

OCT was performed in mydriasis with the Humphrey optical coherence tomography scanner (Humphrey Instruments, Inc, Carl Zeiss, San Leandro, CA, USA). Scanning was performed using an internal fixation beam or an external fixation light for the fellow eye when internal fixation was not possible. Six horizontal scans through the center of the lesion, with a length of $2.83 \mathrm{~mm}$, were performed to provide a good transversal resolution. ${ }^{89}$ Computer controlled, manually placed cursors were used to

Abbreviations: CNV, choroidal neovascularisation; FA, fluorescein angiography; OCT, optical coherence tomography; PDT, photodynamic therapy; RPE, retinal pigment epithelium. 

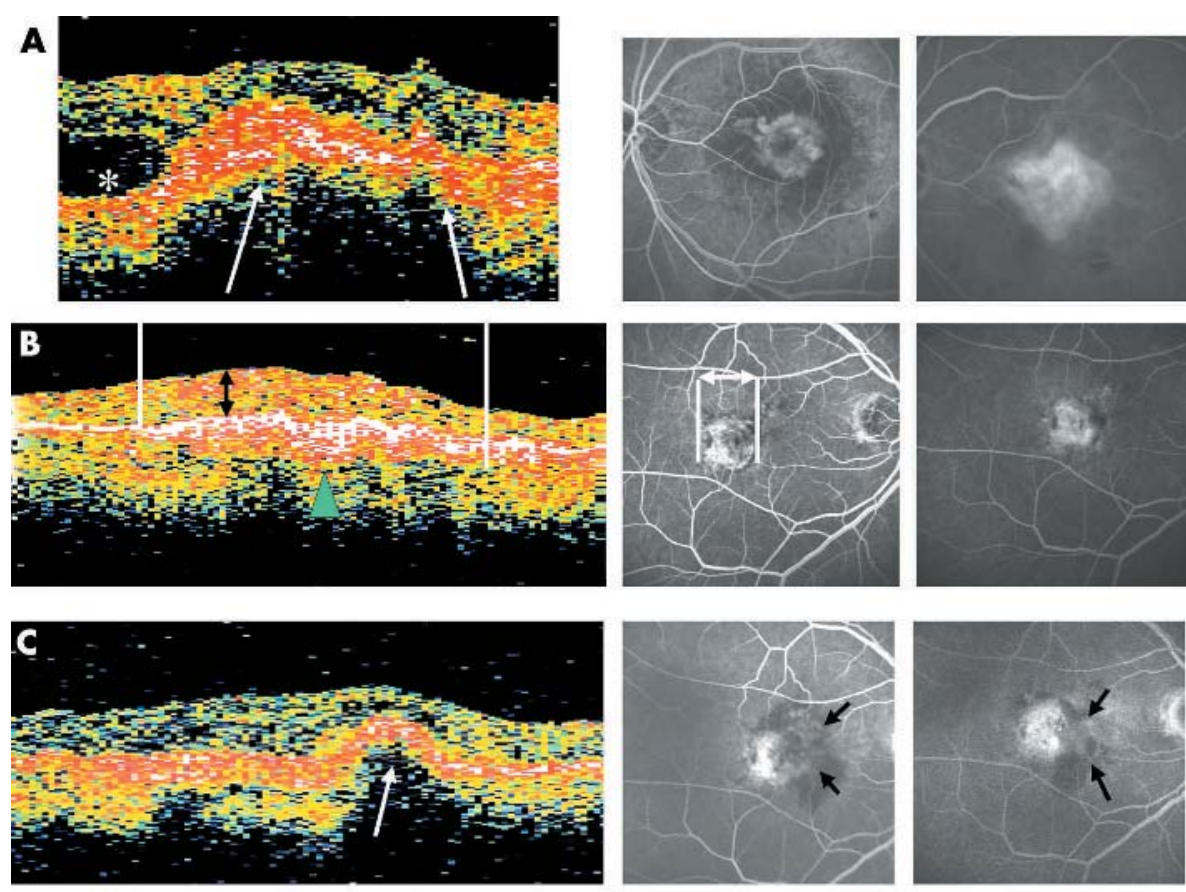

D
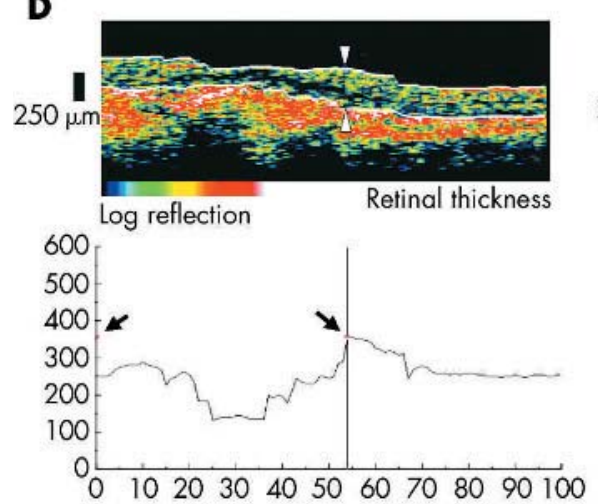
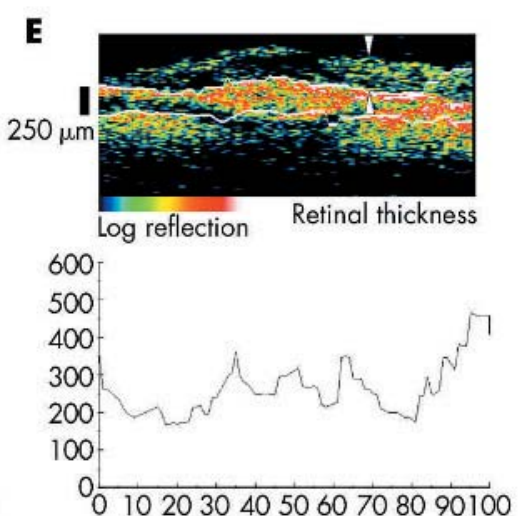

Figure 1 Lines A-C show OCT scans (leff), early phase (middle), and late phase (right) fluorescein angiography images of two of our patients. (A) An active lesion before photodynamic therapy. The reflection from the retinal pigment epithelium (RPE) is thickened fusiformly, elevated, and not well defined beneath (arrows), which is consistent with a detachment of the pigment epithelium. At the nasal side, a detachment of the neurosensory retina is present (asterix). (B) Optical coherence tomography (OCT) and fluorescein angiography (FA) of patient KR. A 75 year old male one year affer the first treatment. He had received four treatments by this time. The arrows show a posterior, not well demarcated (arrow head) thickening of the PE choriocapillaris layer, representing the fibrotic membrane. White lines and arrows demarcate the greatest diameter of the lesion in OCT and FA. There is a partly increased penetration into the tissue. The maximal retinal thickness is $202 \mu \mathrm{m}$ (black arrows). The FA shows well demarcated beginning hyperfluorescence in the early phase with staining in the late phase. The patient was not treated any more. (C) The same eye, with a recurrence, one year later. Up to this time, stable fibrosis was present. White arrows show the elevated, posterior, not well defined, hyperreflectivity with shadowing (haemorrhagic detachment of the pigment epithelium marked by an arrow). The retinal thickness is increased. The reflection from the RPE is thickened. The FA shows blocked fluorescein in the area of the RPE detachment (black arrows). (D and E) The program for retinal thickness measurement was used. (D) The two white lines are positioned correctly, one line demarcating the retinal surface, the other line demarcating the PE. Black arrows show the point of maximum retinal thickness. (E) OCT scans of the same patient are presented. The retinal thickness measuring program is not useful in this case. The neurosensory retina was very hyporeflective and the retinal surface could not be found by the OCT software. Unfortunately, it is not possible to change the position of the lines by the examiner. Manually set cursers have to be used in this case. measure the distance between the highly reflective surface of the retina and the RPE, or the surface of the neovascular membrane corresponding to the retinal thickness. The maximal retinal thickness was measured by two independent examiners (IK and PL). The OCTs, as well as the FAs, were performed by experienced examiners (IK, SB, US).

\section{STATISTICAL METHODS}

The agreement of the two measurements of the maximal retinal thickness were investigated using Bland Altman's plot, the corresponding regression analysis that relates patient wise differences to patient wise means of the two measurements, and Pearson's correlation coefficient. The sensitivity of the maximal retinal thickness was explored statistically by a regression analysis of the successive distance acuity differences at time points 1 to 5 also accounting for an influence of age as well as the correlation between successive distance acuity differences from the same patient (assuming autoregressive residuals). The effect of the treatment on the successive distance acuity difference is analysed by a similar regression analysis with treatment status (period with treatment $v$ period without treatment) as the only covariable. This model was extended by the factor method (WB method) and an interaction term between method and treatment status to see whether the two methods lead to different treatment effects. p Values below 0.05 were understood as statistically significant.

\section{RESULTS}

Forty eyes of 38 patients could be included in the study: 28 eyes participated in subgroup 1 (retreatment based on OCT 


\begin{tabular}{llll} 
Table 1 & \multicolumn{1}{c}{ Demographic data } \\
\hline \multicolumn{5}{c}{ Entire group } & Subgroup 1 & Subgroup 2 \\
\hline Female n, eyes (patients) $20(18)$ & $16(15)$ & 4 \\
Male n, eyes (patients) & 20 & 12 & 8 \\
Age in years (range) & $73(50-88)$ & $74(53-87)$ & 72 \\
Treatments (range) & $2.6(1-4)$ & $2.4(1-4)$ & $4.0(1-5)$ \\
Follow up (range) & $35(24-48)$ & $39(32-48)$ & $29(24-32)$ \\
Mean VA baseline & $0.2(0.1-0.6)$ & $0.2(0.1-0.44)$ & $0.25(0.1-0.6)$ \\
(range) & & & \\
Mean VA month 12 & $0.2(0.08-0.7) 0.2(0.08-0.6)$ & $0.16(0.05-0.7)$ \\
(range) &
\end{tabular}

and FA), and 12 eyes in subgroup 2 (retreatment based on FA only). The demographic data are presented in table 1.

The mean baseline best corrected distance acuity was 0.2 (range 0.1-0.6). These mean values remained unchanged during the first year of follow up. The findings were similar in subgroup one ( 0.2 at baseline and at month 12). In subgroup two the mean values decreased from 0.25 to 0.16 ). After 12 months $37.5 \%$ had a loss of distance acuity of more than three lines in the entire group, $17.8 \%$ in subgroup one, and $66.7 \%$ in subgroup two. The changes of distance acuity over time are presented in figure 2.

The Bland Altman plot shows the agreement between the two measurements (two independent examiners) of the maximum retinal thickness. No statistically significant difference is found in the related regression analysis (95\% confidence interval for mean the intercept is -4.35 to 1.69 and for the slope -0.00059 to 0.175 ). Pearson correlation coefficient between the two measurements is 0.996 .

The mean value of the maximum retinal thickness at baseline was $404.4 \mu \mathrm{m}$ (range 235.0-598.0) and decreased to $281.62 \mu \mathrm{m}$ (range 208.0-458.0) after 12 months. In subgroup one the values decreased from $394.3 \mu \mathrm{m}$ (range 235.0-545.0) to $272.1 \mu \mathrm{m}$ (range 208.0-362.0). In subgroup two the maximum retinal thickness decreased from $428.1 \mu \mathrm{m}$ (range 282.0-598.0) to 303.8 (range 222.0-458.0) The maximum retinal thickness from the OCT does significantly contribute to a prediction of the successive differences of distance acuity at time points 1 to $5(p=0.0041)$. Age $(p=0.6418)$ had no significant influence on the successive distance acuity differences. A slightly higher effect is observed in periods with treatment than in periods without treatment (estimate of difference (standard error) 0.028 (0.015); p value $\mathrm{p}=0.057)$. No significant influence of the method on the treatment effect $(p=0.35)$ is found in the analysis.

The number of treatments was higher (4.0) in subgroup two than in subgroup one (2.4). In subgroup two $18.2 \%$ at

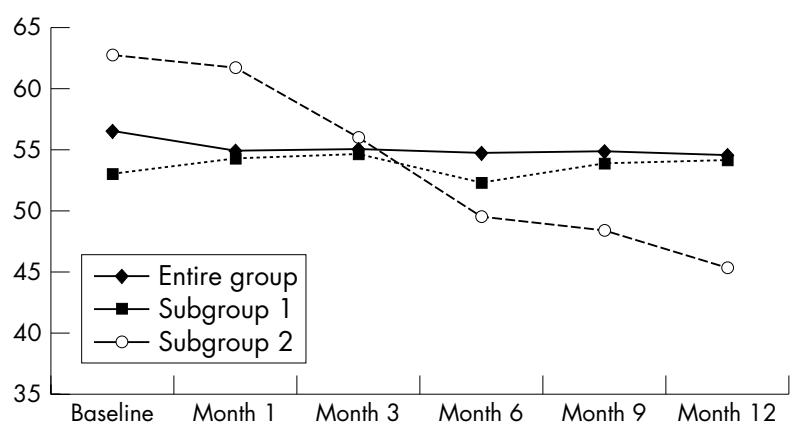

Figure 2 Mean values of the letter scores over time. In the entire group, and in subgroup one (retreatment guided by OCT and FA), the mean values are nearly unchanged at month 12 compared with baseline. In the subgroup two (retreatment based only on FA criteria) there is a decrease of the mean values of the letter scores.

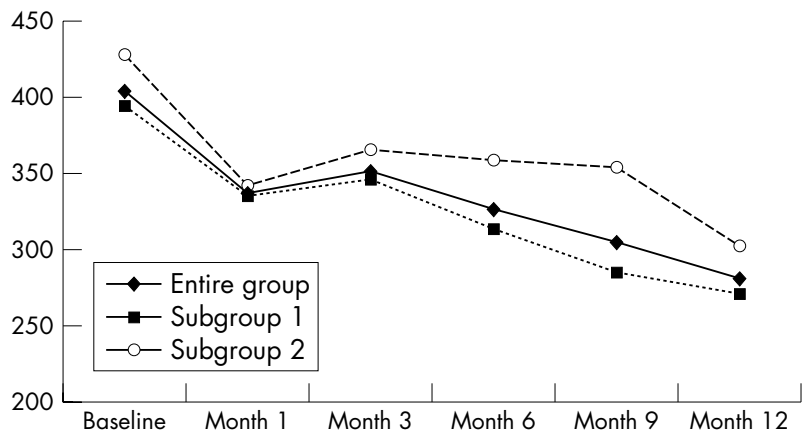

Figure 3 Mean values of the maximum retinal thickness. There is a significant decrease of maximum retinal thickness in all groups.

month $3,27.3 \%$ at month $6,33.3 \%$ at month 9 , and $42.9 \%$ at month 12 would not have been treated including OCT criteria for activity such as in subgroup one. The changes of maximum retinal thickness are presented in figure 3.

\section{DISCUSSION}

The TAP study group has established treatment criteria for PDT based on FA findings. Not only the classification of the type and the composition of the neovascular lesion (classic, occult, and mixed) but also the size of the lesion depend on FA imaging. Recently published studies have shown a considerable variability between different observers interpreting FA images. ${ }^{10}{ }^{11}$ The decision for retreatment depends on the presence of active leakage in the FA. Even after multiple treatments, the fibrovascular tissue remains hyperfluorescent in the late frames. It may be interpreted controversially, whether some active leakage is present or it is only staining. The amount of leakage can only be estimated by the observer in the FA.

The OCT provides objective measurement of the retinal thickness depending on the amount of intraretinal or subretinal fluid accumulations. The changes of retinal thickness have already been used for evaluating the effect of various therapies of diabetic macular oedema. ${ }^{12}{ }^{13}$ or to show the effect of submacular surgery. ${ }^{14}{ }^{15}$ We found a good correlation between the measurements of two independent examiners. There was no statistically significant difference between the measurements. The Pearson correlation coefficient between the two measurements was 0.996. The maximum retinal thickness and the presence of structural changes-such as detachment of the neurosensory retina or detachment of the retinal pigmentepithelium-were used to evaluate the activity of the neovascular lesion. More than $250 \mu \mathrm{m}$ maximum retinal thickness was counted as increased retinal thickness. This corresponds to the data from Pascale Massin et al. ${ }^{16}{ }^{17}$ In normal subjects, mean retinal thickness values are 170 (SE 18) $\mu \mathrm{m}$ for the central zone that is $500 \mu \mathrm{m}$ in diameter and $234(\mathrm{SE} \mathrm{13}) \mu \mathrm{m})$ for the temporal zone of a disc $3000 \mu \mathrm{m}$ in diameter. Rogers et al made a five stage classification of eyes treated with PDT with the help of the OCT. They confirmed the detachment of the neurosensory retina as a sign of activity. ${ }^{18}$

The number of retreatments could be reduced by the help of OCT. The mean number of treatments was 2.4 in subgroup one compared with 4.0 in subgroup two. The distance acuity remained stable in subgroup one and decreased in subgroup two. The changes were not significant, partly because of the differences of the two groups at baseline concerning age, sex, and distance acuity. Although the observation time was at least 36 months in subgroup one, we could observe only one case of a recurrence one year after the fourth treatment (OCT and FA of this patient are presented in lines 2 and 3 of fig 1 ). 
Although PDT is the most selective kind of therapy of CNV we know today, retinal atrophy anterior to the CNV or surrounding it or damage to the RPE may occur in cases treated with PDT. Unnecessary retreatments must be avoided. ${ }^{19}$ PDT is a very expensive therapy. Even if there is no difference in the results of the two treatment strategies one has to avoid unnecessary retreatments. Furthermore, we used the OCT to show the changes of retinal thickness following PDT treatment. There was a significant decrease of retinal thickness at the month 12 examination compared with baseline in both groups. The maximum retinal thickness does contribute significantly to a prediction of the successive differences of distance acuity. Further randomised studies with a higher number of participants will have to provide further evidence that eyes not retreated in cases of inactivity in the OCT reveal at least the same functional results and have no higher risk of recurrences.

When the study was planned (2000), few experiences with the examination of eyes with neovascular diseases of the macular were available. In our study we used relatively short scans with a length of $2.83 \mathrm{~mm}$ to obtain a very good transversal resolution. However the problem of resolution was overemphasised and larger scans would be preferable in many cases (larger lesions). The regimen was not changed later on to obtain comparable data of the follow up examinations. In patients with low vision steady fixation is a problem and the point of fixation changes when the visual acuity decreases. It was not possible to obtain $100 \%$ identical scans at the different examinations. To be able to obtain the maximum retinal thickness multiple scans through the lesion are necessary. The retinal mapping program provides mean values of the retinal thickness of multiple measurements in nine ETDRS areas. ${ }^{20}$ The reproducibility of this kind of measurement of the retinal thickness is very high. Unfortunately this software is not suitable in all cases of neovascular lesions, because the boundary is automatically identified by a threshold algorithm. In some cases the reference point lies at the anterior boundary of the RPE, and in some cases at the anterior boundary of the protruding neovascular membrane (fig 1).

\section{CONCLUSION}

Changes in retinal thickness measured with the OCT can provide evidence of the efficacy of PDT. With the help of OCT it was possible to optimise the retreatment modalities. Fewer treatments might lead to less damage of the retina, therefore improving visual results without increasing the rate of recurrences.

\section{Authors' affiliations}

I Krebs, S Binder, U Stolba, Department of Ophthalmology Rudolf Foundation Clinic, Juchgasse 25, A-1030 Vienna, Austria

I Krebs, S Binder, U Stolba, K Schmid, C Glittenberg, The Ludwig Boltzmann Institute of Retinology and Biomicroscopic Laser Surgery, Vienna, Austria
W Brannath, A Goll, Department of Medical Statistics, University of Vienna, Vienna, Austria

Competing interests: none declared

Ethics approval: the study was approved by the Institutional Review Board of the Rudolf Foundation Clinic. An informed consent was obtained from all patients.

\section{REFERENCES}

1 Treatment of Age-Related Macular Degeneration with Photodynamic Therapy (TAP) Study Group. Photodynamic therapy of subfoveal choroidal neovascularization in age-related macular degeneration with verteporfin: one-year results of 2 randomized clinical trials-TAP report. Arch Ophthalmol 1999; 117:1329-45.

2 Bressler NM, Treatment of Age-Related Macular Degeneration with Photodynamic Therapy (TAP) Study Group. Photodynamic therapy of subfoveal choroidal neovascularization in age-related macular degeneration with verteporfin: two-year results of 2 randomized clinical trials-TAP report 2. Arch Ophthalmol 2001;119:198-207.

3 Krebs I, Binder S, Stolba U, et al. Reading ability and central visual field after photodynamic therapy. Ophthalmologica 2004;218:185-92.

4 Hee MR, Izatt JA, Swanson EA, et al. Optical coherence tomography of the human retina. Arch Ophthalmol 1995;113:325-32.

5 Hee MR, Puliafito CA, Wong C, et al. Quantitative assessment of macular edema with optical coherence tomography. Arch Ophthalmol 1995; 113:1019-29.

6 Macular Photocoagulation Study Group. Subfoveal neovascular lesions in age-related macular degeneration. Guidelines for evaluation and treatment in the macular photocoagulation study. Arch Ophthalmol 1991;109:1242-57.

7 Ferris FL 3rd, Kassoff A, Bresnick GH, et al. New visual acuity charts for clinical research. Am J Ophthalmol 1982;94:91-6.

8 Hee MR, Baumal CR, Puliafito CA, et al. Optical coherence tomography of age-related macular degeneration and choroidal neovascularization. Ophthalmology 1996;103:1260-70.

9 Puliafito CA, Hee MR, Lin CP, et al. Imaging of macular diseases with optical coherence tomography. Ophthalmology 1995;102:217-29.

10 Holz FG, Jorzik J, Schutt F, et al. Agreement among ophthalmologists in evaluating fluorescein angiograms in patients with neovascular age-related macular degeneration for photodynamic therapy eligibility (FLAP-study). Ophthalmology 2003;110:400-5.

11 Friedman SM, Margo CE. Choroidal neovascular membranes: reproducibility of angiographic interpretation. Am J Ophthalmol 2000;130:839-41.

12 Martidis A, Duker JS, Greenberg PB, et al. Intravitreal triamcinolone for refractory diabetic macular edema. Ophthalmology 2002;109:920-7.

13 Massin P, Duguid G, Erginay A, et al. Optical coherence tomography for evaluating diabetic macular edema before and after vitrectomy. Am J Ophthalmol 2003;135:169-77.

14 Zolf R, Glacet-Bernard A, Benhamou N, Imaging analysis with optical coherence tomography: relevance for submacular surgery in high myopia and in multifocal choroiditis, et al. Retina 2002;22:192-201.

15 Brindeau C, Glacet-Bernard A, Coscas F, et al. Surgical removal of subfoveal choroidal neovascularization: visual outcome and prognostic value of fluorescein angiography and optical coherence tomography. Eur J Ophthalmol 2001;11:287-95.

16 Wakitani $Y$, Sasoh M, Sugimoto $M$, et al. Macular thickness measurements in healthy subjects with different axial lengths using optical coherence tomography. Retina 2003;23:177-82.

17 Massin P, Vicaut E, Haouchine B, et al. Reproducibility of retinal mapping using optical coherence tomography. Arch Ophthalmol 2001;119:1135-42.

18 Rogers AH, Martidis A, Greenberg PB, et al. Optical coherence tomography findings following photodynamic therapy of choroidal neovascularization. Am J Ophthalmol 2002;134:566-76.

19 Schmidt-Erfurth U, Michels S, Barbazetto I, et al. Photodynamic effects on choroidal neovascularization and physiological choroid. Invest Ophthalmol Vis Sci 2002;43:830-41.

20 Koozekanani D, Roberts C, Katz SE, et al. Intersession repeatability of macular thickness measurements with the Humphrey 2000 OCT. Invest Ophthalmol Vis Sci 2000;41:1486-91. 